\title{
Catheter ablation of Idiopathic Ventricular Arrhythmias in Nepal-5 Years Single Centre Experience
}

\author{
Roshan Raut ${ }^{1}$, Murari Dhungana ${ }^{1}$, Man Bahadur KC ${ }^{2}$, Mukunda Sharma ${ }^{2}$, Surakshya Joshi ${ }^{1}$, Prashanta \\ Bajracharya ${ }^{1}$, Kunjang Sherpa ${ }^{1}$, Laksheshwor Pradhan${ }^{1}$, Sujeeb Rajbhandari ${ }^{1}$.
}

${ }^{1}$ Department of Cardiology, Shahid Gangalal National Heart Center, Bansbari, Kathmandu, Nepal.

${ }^{2}$ Department of Cardiology, Hospital of advanced medicine and surgery (HAMS), Dhumbarahi, Kathmandu, Nepal.

Corresponding Author: Roshan Raut

Department of Cardiology, Shahid Gangalal National Heart center, Bansbari, Kathmandu, Nepal. Phone: 014370745,9841315902

Email: rautroshan@hotmail.com

ORCID ID NO: 0000-0002-4207-3932

Cite this article as: Raut R., Dhungana M., KC M.B., et al. Catheter ablation of Idiopathic Ventricular Arrhythmias in Nepal-5 Years Single Centre Experience. Nepalese Heart Journal 2021; Vol 18(2), 33-37.

Submission date: $13^{\text {th }}$ July, 2021

Accepted date: $9^{\text {th }}$ October, 2021

Abstract

Background: Idiopathic ventricular arrhythmia (IVAs) is defined as premature ventricular complexes (PVCs), nonsustained
ventricular tachycardia or sustained ventricular tachycardia (VT) in the absence of obvious structural heart disease. Catheter
ablation has become an established treatment strategy for wide varieties of idiopathic ventricular arrhythmias. The aim of this
study is to report the efficacy and safety of catheter ablation of idiopathic ventricular arrhythmias, for the first time in Nepal.
Methods: This is a retrospective observational descriptive study of all patients who underwent electrophysiological study
and radiofrequency catheter ablation for IVAs from March, 2015 to February 2020 at Shahid Gangalal National Heart center
(SGNHC).
Results: Altogether 101 patients underwent an EP study with intent to ablations for idiopathic ventricular arrhythmias. In
13 patients, ventricular arrhythmias were not present on the procedure day and also could not be induced in the lab, therefore
ablation was performed in 88 patients only. RVOT was the most common site of these arrhythmias comprising $51 \%$ of all
cases, followed by fascicular VT (34\%) and basal left ventricular IVAs (15\%). Out of 88 patients, the acute success of 7
patients could not be assessed because of very infrequent PVCs. Out of remaining 81 patients, acute success achieved in 77
patients (95\%). Recurrence occurred in 9 patients (10.7\%) and 4 patients underwent repeat ablation giving rise to over clinical
success during follow up in 78 patients $(88.7 \%)$. There were two major complications, one pulmonary embolism and another
cardiac tamponade both managed successfully. Conclusion: This single-center single operator study demonstrates that catheter ablation of idiopathic ventricular arrhythmias has a high success and low complication rate.

Keywords: Idiopathic ventricular arrhythmias, Right ventricular outflow tract, Fascicular Ventricular tachycardia, Left ventricular outflow tract

DOI: https://doi.org/10.3126/njh.v18i2.40402

\section{Introduction}

Idiopathic ventricular arrhythmia (IVAs) is defined as premature ventricular complexes (PVCs), nonsustained ventricular tachycardia or sustained ventricular tachycardia (VT) in the absence of obvious structural heart disease ${ }^{1,2}$. IVAs can occasionally occur in the presence of structural heart disease, but it is not related to the ventricular substrate. IVAs most commonly occur from outflow tract, and right ventricular outflow tract origin (RVOT) is more common than left ventricular outflow tract (LVOT). ${ }^{1,3}$ In LVOT, the most common site of origin is aortic root, followed by other structures like aortomitral continuity, mitral annulus and epicardial origin like LV summit.4 IVAs can arise from the Purkinje network, most commonly from the left posterior fascicle followed by the anterior, septal fascicles and papillary muscles ${ }^{5,6}$. Usually IVAs, in the absence of heart disease, are benign with good long term prognosis 7 . However some patients are highly symptomatic and warrant treatment ${ }^{8}$. It has also been recognized that frequent ventricular ectopics, if not treated, may lead to reduction in LV function, called PVC induced cardiomyopathy ${ }^{8}$. A study indicates that in patients with PVCs cardiomyopathy, the median PVCs burden was $24 \%$ but it can be as low as $10 \%{ }^{9}$. Antiarrhythmic medicines can be unsuccessful in many patients due to lower efficacy and intolerance ${ }^{10}$. Therefore, catheter ablation has become an established treatment strategy for wide varieties of idiopathic ventricular arrhythmias (IVAs). Catheter ablation is recommended in patients with symptomatic ventricular arrhythmias if antiarrhythmic medical therapy has been failed or not been tolerated, or if patient does not want medication or if there is development of PVCs induced cardiomyopathy ${ }^{11}$. Moreover, catheter ablation is preferable to antiarrhythmic medicine in PVCs from RVOT because of very high success rate of $80-95 \%$ with low complication rate ${ }^{12-14}$. Similarly, ablation of idiopathic sustained VT is also preferable to medicine as being more definite treatment with 
high efficacy and low complication rate ${ }^{12,15}$. In a multicenter cohort study, it was found that the only significant predictor of continued clinical success during follow up was RVOT PVCs location ${ }^{16}$.

There is no knowledge and published data regarding catheter ablation of IVAs in Nepal. Since 2015, we have been routinely performing catheter ablation of idiopathic VAs which led us an opportunity to report a single centre experience on the outcome of catheter ablation of IVAs in Nepal. This study will provide the insights from the early experience of catheter ablation of IVAs.

\section{Methods}

Patient population: This is a retrospective observational descriptive study of all consecutive patients who underwent electrophysiological study and radiofrequency catheter ablation for IVAs from March, 2015 to February 2020 at Shahid Gangalal National Heart center (SGNHC). A single primary operator was involved in the ablations. The data was collected from the procedural notes, the discharge notes and electrophysiology log books. A proforma of each patient was filled up with the collected data. The data was then analyzed to evaluate the baseline characteristics, the immediate outcome including non-inducible ventricular arrhythmias in the lab, the acute success, the acute success not clear due to very infrequent PVCs, and the complications. The follow up outcome included the recurrences, the repeat ablations and the clinical success. The baseline characteristics included age, sex, left ventricular ejection fraction (LVEF), types of ventricular arrhythmias like premature ventricular complexes or non-sustained/sustained ventricular tachycardia, localization of ventricular arrhythmias, types and use of 3D mapping system. The presence of structural heart disease was evaluated by 12 lead electrocardiogram, transthoracic echocardiogram, and exercise stress test if deemed necessary. Coronary angiogram and cardiac magnetic resonance imaging (MRI) were occasionally done if coronary artery disease or scar related ventricular arrhythmias(VA) were suspected. The site of VAs origin was categorized as RVOT, LV base (Aortic root, Aortomitral continuity, mitral annulus, LV summit) and left fascicles (anterior and posterior fascicles) based on successful ablation site. Successful ablation of ventricular arrhythmias was defined as termination of sustained VT or elimination of PVCs/NSVT during ablation with no recurrence at least 30 mins after the last ablation. The follow up outcome was assessed at one month, 6 months to 1 year, and longer if deemed necessary. For evaluating the outcome, holter was done at least once during follow up and repeated if deemed necessary. The clinical success was defined as no recurrence of VT or at least $80 \%$ decrease in PVCs burden after first or repeat ablation. 16

\section{Inclusion criteria:}

1. Symptomatic PVCs with failed antiarrhythmic medicine

2. Symptomatic PVCs with intolerable to antiarrhythmic medicine.

3. Symptomatic PVCs with patient preference in favor of ablations.

4. Suspected PVC- induced cardiomyopathy.

Exclusion criteria: VAs suspected due to structural heart disease.

Electrophysiological study and catheter ablations: In general, the procedure was done as follows. Four multipolar electrode catheters were placed in the high right atrium, the His bundle region, the right ventricle and the coronary sinus, respectively for the recording of endocardial electrograms and programmed stimulation. If there were no PVCs or VTs at baseline, they were induced by burst pacing or programmed stimulation from the RV apex or RVOT. If still not inducible, protocols were repeated with isoprenaline infusion. The ablation of initial few cases were done conventionally without $3 \mathrm{D}$ mapping system, which was not available in Nepal at that time. The EnSite NavX system (St Jude Medical, St Paul, Minnesota, USA) was used for 3D mapping in rest of the cases. Electroanatomical mapping was done with the ablation catheter (usually $4 \mathrm{~mm}$ tip 6/7 Fr nonirrigated catheter). Activation mapping was primarily performed to find the earliest site and complimented with pace mapping. When activation mapping was not possible because of very infrequent VAs, pace mapping was done to find the target ablation site. Ablation of right VAs was primarily done with $4 \mathrm{~mm}$ tip 6 or 7 Fr non-irrigated catheters(St jude medical or J \& J) whereas that of left VAs with the $4 \mathrm{~mm}$ tip 7 Fr Cool Flex catheter (St. Jude Medical, St. Paul, MA, USA). For the right sided procedure, intravenous bolus of heparin 3000 units was given without ACT monitoring, where as for the left sided procedure, bolus 3000 heparin with additional dose to keep ACT 250-300 seconds was given.

Statistical Analysis: Continuous variable was expressed as mean \pm standard deviation or median and interquartile range if data is skewed. Categorical variable was expressed in percentage. SPSS software was used for statistical analysis. This study was approved by the Institutional Review Board of SGNHC.

\section{Results}

From March 2015 till February, 2020, altogether 101 patients underwent an EP study of idiopathic ventricular arrhythmias with intent to ablations. In 13 patients, ventricular arrhythmias were not present on the procedure day and could not be induced in the lab, therefore, ablation was performed in 88 patients only. The baseline characteristics are shown in table 1.

Table 1: Baseline characteristics

\begin{tabular}{|l|l|}
\hline \multicolumn{1}{|c|}{ Patients(No) } & \multicolumn{1}{|c|}{101} \\
\hline Age $($ yrs in mean \pm SD) & $38 \pm 14$ \\
\hline Age(yrs in range) & 7 to 77 \\
\hline Male(\%) & $50 / 101(49.5 \%)$ \\
\hline LVEF $<50 \%(\%)$ & $8 / 90(8.8 \%)$ \\
\hline Sustained VT & $36 / 101(35.6 \%)$ \\
\hline NSVT(\%) & $10 / 101(9.9 \%)$ \\
\hline PVCs(\%) & $55 / 101(54.5 \%)$ \\
\hline $\begin{array}{l}\text { Ventricular arrhythmias present } \\
\text { or was inducible for mapping }\end{array}$ & $88 / 101(87 \%)$ \\
\hline Suspected IVAs induced & $8 / 90(8.8 \%)$ \\
\hline cardiomyopathy & \\
\hline Site of origin of VAs & $45 / 88(51.1 \%)$ \\
\hline RVOT & $13 / 88(14.8 \%)$ \\
\hline LV Base & $30 / 88(34.1 \%)$ \\
\hline Fascicular VT & \\
\hline
\end{tabular}

Out of 88 patients, 7 patients had very infrequent PVCs at baseline, even with isoprenaline infusion. In these patients, ablation was done based on pace map only, and thereby acute success could not be assessed at the end of the procedure. For remaining 81 patients, there was a reasonable frequency of VAs for activation mapping and acute success could be assessed. The outcome data has been shown in table 2 . 
Table 2: Outcome data: Main Findings

\begin{tabular}{|c|c|}
\hline $\begin{array}{l}\text { Acute success could not be } \\
\text { assessed }\end{array}$ & $7 / 88(7.9 \%)$ \\
\hline Overall acute success & $77 / 81(95 \%)$ \\
\hline $\begin{array}{l}\text { Recurrence in patients who had } \\
\text { acute success }\end{array}$ & $6 / 77(7.7 \%)$ \\
\hline $\begin{array}{l}\text { Recurrence in patients whose } \\
\text { acute success could not be } \\
\text { assessed }\end{array}$ & $3 / 7(42.8 \%)$ \\
\hline Overall recurrence & $9 / 84(10.7 \%)$ \\
\hline Repeat ablations & 4 \\
\hline Successful after repeat ablation & $3 / 4$ \\
\hline $\begin{array}{l}\text { Overall clinical success during } \\
\text { follow up }\end{array}$ & $78 / 88(88.6 \%)$ \\
\hline $\begin{array}{l}\text { Improvement of LV function in } \\
\text { IVAs induced cardiomyopathy }\end{array}$ & $6 / 8(75 \%)$ \\
\hline Complications & $2 / 101(1.9 \%)$ \\
\hline Pericardial tamponade & 1 \\
\hline Pulmonary embolism & 1 \\
\hline Mortality & 0 \\
\hline
\end{tabular}

Table 3: Outcome data based on different locations

\begin{tabular}{|c|c|c|c|}
\hline & \multicolumn{3}{|c|}{ Locations } \\
\hline & $\begin{array}{l}\text { RVOT } \\
\text { region }\end{array}$ & $\begin{array}{l}\text { LV basal } \\
\text { region }\end{array}$ & $\begin{array}{c}\text { Fascicular } \\
\text { VT }\end{array}$ \\
\hline No. of patients & 45 & 13 & 30 \\
\hline $\begin{array}{l}\text { LV dysfunction } \\
(\mathrm{LVEF}<50 \%)\end{array}$ & 5 & 1 & 2 \\
\hline Mean age (Years) & $41 \pm 14$ & $40 \pm 20$ & $29 \pm 11$ \\
\hline Male & $16(35.5 \%)$ & $8(61 \%)$ & $23(76.6 \%)$ \\
\hline $\begin{array}{l}\text { Acute success could not } \\
\text { be assessed }\end{array}$ & 6 & 1 & 0 \\
\hline Acute success & $\begin{array}{l}38 / 39 \\
(97.4 \%)\end{array}$ & $\begin{array}{l}10 / 12 \\
(83.3 \%)\end{array}$ & $29(96.6 \%)$ \\
\hline Recurrence & $5(11.3 \%)$ & $1(9 \%)$ & $3(10 \%)$ \\
\hline $\begin{array}{l}\text { Successful after Repeat } \\
\text { ablations }\end{array}$ & $2 / 2$ & $0 / 1$ & $1 / 1$ \\
\hline Overall clinical success & $41(91 \%)$ & $10(76.9 \%)$ & $27(90 \%)$ \\
\hline Complication & & & 2 \\
\hline
\end{tabular}

Table 4: Location of Ventricular arrhythmias

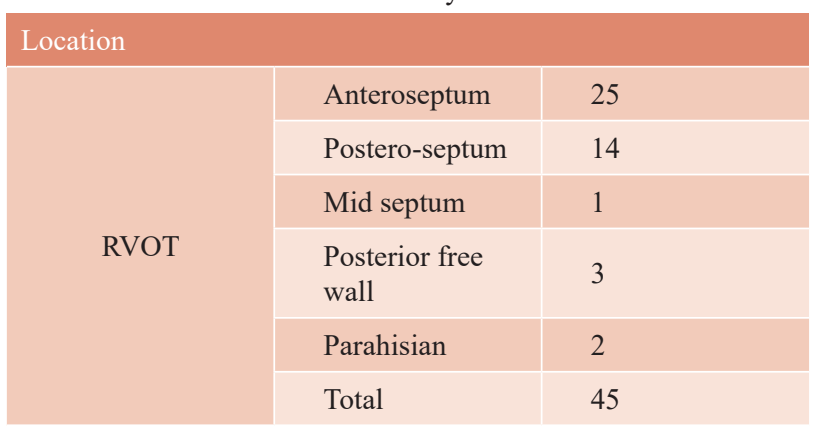

\begin{tabular}{|l|l|l|}
\hline \multirow{2}{*}{ LV basal } & Aortic cusps & 5 \\
\hline & $\begin{array}{l}\text { Aortomitral } \\
\text { continuity }\end{array}$ & 3 \\
\hline & LV summit & 3 \\
\hline & Mitral Annulus & 1 \\
\hline & $\begin{array}{l}\text { Bundle branch } \\
\text { reentry VT }\end{array}$ & 1 \\
\hline & Total & 13 \\
\hline \multirow{3}{*}{ Fascicular VT } & $\begin{array}{l}\text { Posterior } \\
\text { Fascicle }\end{array}$ & 28 \\
\hline & Anterior Fascicle & 2 \\
\hline & Total & 30 \\
\hline
\end{tabular}

\section{Discussion}

Baseline Characteristics: The most common clinical presentation of IVAs was premature ventricular complexes (PVCs) being $54 \%$. RVOT was the most common site of these arrhythmias comprising $51 \%$ of all cases. Our findings are consistent with the previous studies ${ }^{13,17}$. RVOT IVAs were more common in female patients $(64.5 \%)$ whereas fascicular VT were more common in male patients $(76 \%)$. There was more or less equal distribution of male and female patients in case of LV basal IVAs (8 male and 6 female). Patients with fascicular VT were relatively younger compared to RVOT and LV basal IVAs (Mean age: 29 vs 41/40 years). Our report is consistent with the previous studies which shows that gender and age difference exists between different IVAs ${ }^{18}$.

Main findings: In 7 patients, acute success could not be assessed due to very infrequent PVCs. Acute success was assessed in remaining 81 patients out of which 77 patients achieved acute success $(95 \%)$. Out of 77 cases with acute success, recurrence occurred in 6 patients $(7.7 \%)$, whereas in other 7 patients whose acute success could not be assessed, recurrence occurred in 3 patients $(44.8 \%)$, overall recurrence being $10.7 \%$. Repeat ablation was performed in 4 patients out of which successful ablation was achieved in 3 patients. Thus, the overall clinical success after repeat ablations during follow up period was $88.6 \%$. Our result is comparable to a large multicentre study which showed their acute success rate of $84 \%$ and clinical success rate of $71 \% .{ }^{16}$ We have data of baseline echo in 90 patients only, and IVAs induced cardiomyopathy was found in 8 patients $(8.8 \%)$ out of which 6 patients $(75 \%)$ had improvement in LV function after successful ablation. Clinical studies have reported the diagnosis of PVCs induced cardiomyopathy in $9 \%-30 \%$ of patients referred for PVC ablation ${ }^{9,16,19}$. Prevalence of IVAs induced cardiomyopathy in our patient population seems less than the other studies, likely because of skewed data due to small sample size, missed echo data and referral bias. Moreover, we have 2 patients with incessant fascicular VT who presented with heart failure with severe LV systolic dysfunction, both of them had full recovery of LV function after successful ablation. Interestingly, both patients were children, age being 7 and 9 years respectively. Automated atrial tachycardia ${ }^{20}$ and permanent junctional reciprocating tachycardia ${ }^{21}$ has been well known to be the common cause of tachycardia induced cardiomyopathy in children, however incessant fascicular VT leading to cardiomyopathy has been rarely reported in the literature ${ }^{22-24}$. Little is known about the pathophysiology of incessant nature of tachycardia. A focal fibrosis not related with ischemia or myocarditis in close proximity to the left posterior fascicle was presumed to be the possible cause of the incessant behavior in a few isolated case of fascicular VT ${ }^{25}$. 
There were 2 major complication observed, both in patients with fascicular VT ablation, one was pulmonary embolism which recovered after the conservative management and another pericardial tamponade that required emergency pericardiocentesis. There was no mortality in our case series.

Outcome based on different locations: The success rate of ablation seems to be better in RVOT IVAs and fascicular VT as compared to LV basal IVAS; Acute success was $97 \%$ for RVOT and $96.6 \%$ for Fascicular VT whereas only $83.3 \%$ for LV basal IVAs. Similarly, the overall clinical success during follow up was $91 \%$ for RVOT, $90 \%$ for fascicular VT and only $76.9 \%$ for LV basal IVAs. Our report is similar to several other studies which showed that RVOT IVAs and Fascicular VT has better ablation outcome compared to LV basal IVAs. ${ }^{12,16}$

Location of ventricular arrhythmias: In RVOT, the most common site was antero-septal RVOT (55\%), followed by posteroseptum (31\%). In LV basal IVAs, most common site was aortic cusps followed by aortomitral continuity and LV summit. In Fascicular VT, posterior fascicle was more common than anterior fascicle $(93 \%$ vs 7\%). Our report is similar to several previous studies. ${ }^{3,4,5,6}$

\section{Limitation}

1. This is a single center single operator study.

2. We do not have data of the antiarrhythmic medicine used.

3. We also do not have data on amount of PVC burden to correlate with the PVCS induced cardiomyopathy.

4. Due to small number of patients in LV basal groups, the findings might not be very reliable due to skewed data.

5. Our study is basically an observational descriptive study. Comparison between different site of ventricular arrhythmias with regards to age, gender and outcome using statistical significance test is out of scope of this study.

\section{Conclusion}

This single-center single operator study demonstrates that catheter ablation of idiopathic ventricular arrhythmias has a high success and low complication rate.

\section{References}

1. Lerman BB: Ventricular tachycardia in patients with structurally normal hearts. In Zipes DP, Jalife J, editors: Cardiac electrophysiology: from cell to bedside, ed 5, Philadelphia, 2009, WB Saunders, pp 657-668. https://doi. org/10.1016/B0-7216-0323-8/50075-0

2. Stevenson WG, Soejima K. Catheter ablation for ventricular tachycardia. Circulation. 2007;115:2750-2760. https://doi. org/10.1161/CIRCULATIONAHA.106.655720

3. Yamada T, Kay GN. How to diagnose and ablate ventricular tachycardia from the outflow tract and aortic cusps. In: AlAhmad A, Callans DJ, Hsia HH, Natale A, Oseroff O, Wang PJ, editors. Hands-On Ablation: The Experts' Approach. 2nd ed. Minneapolis, MN: Cardiotext Publishing; 2017. pp. 292301

4. Natale A, Raviele A, Al-Ahmad A, et al: Venice Chart International Consensus document on ventricular tachycardia/ ventricular "brillation ablation, J Cardiovasc Electrophysiol 21:339-379,2010. https://doi.org/10.1111/j.15408167.2009.01686.x

5. Nogami A, Naito S, Tada H, Taniguchi K, Okamoto $Y$, Nishimura S, Yamauchi Y,Aonuma K, Goya M, Iesaka Y, Hiroe M. Demonstration of diastolic and presystolic Purkinje potentials as critical potentials in a macroreentry circuit of verapamil-sensitiveidiopathic left ventricular tachycardia. Journal of the American College of Cardiology.2000;36:811-823. https://doi.org/10.1016/S07351097(00)00780-4

6. Yamada T, Doppalapudi H, McElderry HT, Okada T, Murakami Y, Inden Y, Yoshida Y, Kaneko S, Yoshida N, Murohara T, Epstein AE, Plumb VJ, Kay GN. Idiopathic ventricular arrhythmias originating from the papillary muscles in the left ventricle: Prevalence, electrocardiographic and electrophysiological characteristics, and results of the radiofrequency catheter ablation. Journal of Cardiovascular Electrophysiology. 2010;21:62-69 https://doi.org/10.1111/ j.1540-8167.2009.01594.x

7. Kennedy HL, Whitlock JA, Sprague MK, et al. Long-term followup of asymptomatic healthy subjects with frequent and complex ventricular ectopy. N Engl J Med. 1985;312:193-7. https://doi.org/10.1056/NEJM198501243120401

8. Bogun F, Crawford T, Reich S, et al. Radiofrequency ablation of frequent, idiopathic premature ventricular complexes: comparison with a control group without intervention. Heart Rhythm. 2007;4:863-7. https://doi.org/10.1016/j. hrthm.2007.03.003

9. Baman TS, Lange DC, Ilg KJ, Gupta SK, Liu TY, Alguire C, Armstrong W, Good E, Chugh A, Jongnarangsin K, Pelosi F Jr, Crawford T, Ebinger M, Oral H, Morady F, Bogun F.Heart Rhythm. 2010 Jul;7(7):865-9. https://doi.org/10.1016/j. hrthm.2010.03.036

10. Ling Z, Liu Z, Su L, et al. Radiofrequency ablation versus antiarrhythmic medication for treatment of ventricular premature beats from the right ventricular outflow tract: prospective randomizedstudy. Circ Arrhythm Electrophysiol. 2014;7:237-43. https://doi.org/10.1161/CIRCEP.113.000805

11. Priori SG, Blomström-Lundqvist C, Mazzanti A, et al. ESC Guidelines for the management of patients with ventricular arrhythmias and the prevention of sudden cardiac death. Eur Heart J.2015;36:2793-867. https://doi.org/10.1093/eurheartj/ ehv316

12. $2019 \mathrm{HRS} / \mathrm{EHRA} / \mathrm{APHRS} / \mathrm{LAHRS}$ expert consensus statement on catheter ablation of ventricular arrhythmias

13. Ling Z, Liu Z, Su L, et al. Radiofrequency ablation versus antiarrhythmic medication for treatment of ventricular premature beats from the right ventricular outflow tract: prospective randomized study. Circ Arrhythm Electrophysiol 2014;7:237-243. https://doi.org/10.1161/CIRCEP.113.000805

14. Lamba J, Redfearn DP, Michael KA, Simpson CS, Abdollah H, Baranchuk A. Radiofrequency catheter ablation for the treatment of idiopathic premature ventricular contractions originating from the right ventriculal outflow tract: a systematic review and meta-analysis. Pacing Clin Electrophysiol 2014;37:73-78. https://doi.org/10.1111/ pace. 12243

15. Coggins DL, Lee RJ, Sweeney J, et al. Radiofrequency catheter ablation as a cure for idiopathic tachycardia of both left and right ventricular origin. J Am Coll Cardiol 1994;23:1333-1341. https://doi.org/10.1016/07351097(94)90375

16. Latchamsetty R, Yokokawa M, Morady F, et al. Multicenter outcomes for catheter ablation of idiopathic premature ventricular complexes. JACC Clin Electrophysiol 2015;1:116123. https://doi.org/10.1016/j.jacep.2015.04.005 
17. Surksha Sirichand, MD et al. Incidence of Idiopathic Ventricular Arrhythmias: A Population Based Study. Circ Arrhythm Electrophysiol. 2017 February ; 10(2) https://doi. org/10.1161/CIRCEP.116.004662

18. Mikiko Nakagawa et al. Gender differences in various types of idiopathic ventricular tachycardia. J Cardiovasc Electrophysiol $2002 \mathrm{Jul} ; 13(7): 633-8$.

19. Yokokawa M, Good E, Crawford T et al. Recovery from left ventricular dysfunction after ablation of frequent premature ventricular complexes. Heart Rhythm 2013;10:172-5. https:// doi.org/10.1016/j.hrthm.2012.10.011

20. R.E. Velázquez, E.A. Martínez. Dilated cardiomyopathy induced by ectopic atrial tachycardia. Arch Inst Cardiol Mex, 70 (2000), pp. 292-300

21. P. Noë, V. Van Driel, F. Wittkampf, et al.Rapid recovery of cardiac function after catheter ablation of persistent junctional reciprocating tachycardia in children.Pacing Clin Electrophysiol, 25 (2002), pp. 191-194. https://doi. org/10.1046/j.1460-9592.2002.00191.x

22. B. Singh, U. Kaul, K.K. Talwar, et al.Reversibility of tachycardia induced cardiomyopathy following the cure of idiopathic left ventricular tachycardia using radiofrequency energy.Pacing Clin Electrophysiol, 19 (1996), pp. 1391-1392 https://doi.org/10.1111/j.1540-8159.1996.tb04222.x

23. A. Arya, M. Haghjoo, P. Davari, et al.Resolution of tachycardia-induced cardiomyopathy following ablation of verapamil-sensitive idiopathic left ventricular tachycardia. Pediatr Cardiol, 27 (2006), pp. 146-148. https://doi. org/10.1007/s00246-005-1091-4

24. P. De Filippo, P. Ferrero, R. Brambilla, et al. Curative ablation in a 12-month-old baby with severe ventricular systolic dysfunction and incessant ventricular tachycardia. Europace, 13 (2011), pp. 1052-1055. https://doi.org/10.1093/europace/ eur054

25. D-W. Lee, I-Y. Oh, J-W. Seo, et al. A case of focal fibrosis near the fascicle as a possible cause of incessant ventricular tachycardia.Pacing Clin Electrophysiol, 35 (2012), pp. e131-e135. https://doi.org/10.1111/j.1540-8159.2011.03140.x 